\title{
Saliva AND ORAL HeALTH
}

The properties and functions of saliva have been studied extensively for more than sixty years. This complex biofluid plays an essential role in the maintenance of oral health. Saliva is constituted by water, organic and inorganic components which have biological functions essential for homeostasis of the oral cavity. It contains a wide variety of unique proteins, including prolinerich proteins (PRPs) and enzymes such as lysozyme, lactoferrin, peroxydases, and secretory IgAs. Saliva secretion is controlled by the autonomous nervous system with the volume produced varying according to the type and intensity of stimulation. A greater volume is produced before, during and after meals whereas a lower salivary production is observed during sleep.. Adequate salivary flow and composition are recognized as important for lubrication and protection of soft and hard oral tissues. Protection of soft tissues is provided against desiccation, penetration, ulceration, and potential carcinogens by mucin and anti-proteases. A major protective function results from the salivary role in stabilizing the ecological balance in the oral cavity via clearance, aggregation and reduced adherence by both immunological and non-immunological means as well as direct antimicrobial activity. Saliva is effective in maintaining $\mathrm{pH}$ in the oral cavity by its buffer capacity and contributes to the regulation of dental biofilm $\mathrm{pH}$ and enamel surface integrity. Salivary maintenance of tooth integrity depends on mechanical cleansing and enamel remineralization ${ }^{1,2}$. For these reasons individuals with impaired salivary synthesis and secretion may have difficulties in eating, swallowing and become prone to oral diseases such as mucosal infections and dental caries.

In recent years, in addition to the studies referring to the role of saliva in health and disease, major efforts have been made to better understand the composition of whole human saliva and its individual variations. A better comprehension of saliva biomarkers would allow use as a non-invasive diagnostic tool. These studies including proteomic and transcriptomic approaches appear to be promising for early detection of several oral and systemic chronic diseases 3,4 .

In the past decade, significant effort has led to advances in revealing the etiological links between chronic inflammatory dental disease and systemic conditions ${ }^{5}$. These studies mainly suggest an association between periodontal health and cardiovascular disease, diabetes mellitus and pre-term birth and low birth-weight neonates ${ }^{6}$. Scarce information is available concerning the effect of overweight and obesity on oral health. Further, a few studies have suggested that obesity can affect periodontal diseases and vice-versa ${ }^{7,8}$. However, at present, the effect of this worldwide epidemic, on children's oral health requires elucidation.

In the present issue, Pannunzio et al, analyze stimulated whole saliva in overweight and obese schoolchildren in the city of Bragança Paulista, State of Sao Paulo, Brazil ${ }^{9}$. This original study is of particular relevance considering that scientific evidence has shown that increased body fat mass during childhood and adolescence affects general health ${ }^{10}$.
This cross-sectional study determined the salivary $\mathrm{pH}$, flow rate, buffer capacity, protein content, phosphate, calcium, fluoride, total and free sialic acid, and peroxidase activity in three groups of schoolchildren (overweight, obese and control) 7 to 10 years of age. Comparative analysis of saliva parameters between groups showed that overweight and obese children had alterations of phosphate, free sialic acid and proteins concentrations in the whole saliva samples, whereas no statistically significant differences were observed in saliva flow rate, calcium and fluoride concentrations and saliva buffer capacity. The overweight and obese children, when compared with the control group had lower phosphate concentration, higher free sialic acid content, higher protein content and reduced peroxidase activity. As suggested by the authors, the altered parameters are favorable conditions for development of dental caries. Further studies would be of interest to verify if the saliva changes observed are correlated with an increase of dental carie activity in the overweight and obese children. Also, a characterization of the increased proteins would be useful to better understand which salivary proteins increase and if aggregation/ adherence properties are altered in these samples. Especially since recent studies have suggested that levels of salivary proteins such as statherin and truncated cystatin S may be potential risk indicators for development of caries and other oral diseases ${ }^{11}$.

Ana Carolina Acevedo

Adjunt Professor, Department of Dentistry, Health Science Faculty, University of Brasilia, Brasilia, Brazil.

acevpoppe@gmail.com

\section{References}

1. Mandel ID. Functions of saliva. J Dent Res.1987;66:623-7.

2.Wolff MS, Larson C. The cariogenic dental biofilm: good, bad or just something to control? Braz Oral Res. 2009;1:31-8.

3.Segal A,Wong DT. Salivary diagnostics: enhancing diseases detection and making medicine better. Eur J Dent Educ. 2008;12(Suppl 1):22-9.

4.Giannobile WV, Beikler T, Kinney JS, Ramseir CA, Morelli, Wong DT. Saliva as a diagnostic tool for periodontal disease: current state and future directions. Periodontology 2009;30:52-64.

5. Reddy MS. Reaching a better understanding of non-oral disease and implication of periodontal infections. Periodontology. 2000. 2007;44:9-14.

6.Philstrom BL,MIchalowicz, Johnson NW. Periodontal diseases. Lancet. 2005;366:1809-20.

7.Ritchie CS. Obesity and periodontal disease. Periodontology 2000. 2007;44:154-65.

8.Goodson JM,Groppo D, Halem S, Corpino E. Is obesity an oral bacterial disease? J Dent Res. 2009;88:519-23.

9. Ebbeling CB, Pawlak DB, Ludwig DS. Childhood obesity: public-health crisis, comom sense cure. The Lancet. 2002;360:473-82.

10. Rudney JD, Staikov RK, Johnson JD. Potential biomarkers of human salivary function: a modified proteomic approach. Arch Oral Biol. 2009;54:91-100.

11. Pannuzio E, Amancio OMS, Vitalle MSS, Souza DN, Mendes FM, Nicolau $\mathrm{J}$. Analysis of the stimulated whole saliva in overweight and obese school children. Rev Assoc Med Bras. 2010; 56(1) 32-36. 